\title{
Blood Transfusion during caesarean delivery
}

\author{
Premarathna HKSI, Rangana GWAR, Weerasinghe CJ, Wicramanayaka RP, Jayaweera MTD
}

\begin{abstract}
Introduction: Blood transfusion is considered as a life saving measure in obstetrics. However, at present it is not considered safe all the time due to transfusion reactions and infections. The mother is at risk of increased bleeding, because caesarean section may result in twice the blood loss of vaginal delivery (due to placental attachment and two surgical scars). In Sri Lankan setup, grouping and cross matching are done prior to each and every caesarean section without considering the presence or absence of risk factors even with less facilities available. During caesarean section in Sri Lanka blood transfusion to cross matching ratio is considered to be low.
\end{abstract}

Objectives: To determine the proportion of blood transfusions performed among mothers whose blood was grouped and cross-matched for Lower segment caesarean section (LSCS). To find out the indications/ associated factors for blood transfusion in LSCS

Method \& Materials: A retrospective cross sectional study carried out in Obstetric Units, General Hospital Matara Sri Lanka. Data collected from all the bed head tickets and other records available at Blood Bank General Hospital Matara belong to mothers who underwent elective and emergency caesarean sections from $30^{\text {th }}$ July 2013 to $1^{\text {st }}$ of June 2014

Results: Our sample size was 3174 mothers majority underwent Elective Lower segment caesarean section (EL/LSCS) $(57.5 \%)$ \& 42.5\% were Emergency Lower segment caesarean section (EM/LSCS). Only $1.85 \%$ of mothers transfused blood during LSCS, as $3.38 \%$ during EL/LSCS \& 96.6\% during EM/LSCS. Lower segment large fibroid (50\%) \& placenta accreta + past section (50\%) were two indications that need blood transfusion during EL/LSCS in our research. Antepartum haemorrhage (APH) (15.78\%), pregnancy induced hypertension (PIH) (8.77\%), postpartum haemorrhage $(26.31 \%)$, Anaemia $\mathrm{Hb} 7.6-8.2 \mathrm{~g} / \mathrm{dl}$ (36.84\%), Twin pregnancy (5.26\%) \& placenta previa were other indication that need blood transfusion in EM/LSCS.

Conclusions: This study shows each \& every mother who undergo EL/LSCS do not need blood transfusion \& cross matching especially in EL/LSCS. According to our study

\section{District General Hospital Matara}

patients with following risk factors need blood grouping $\&$ cross matching. APH, PIH, Placental abnormalities, Anaemia ( $\mathrm{Hb} \quad 7.6-8.2 \mathrm{~g} / \mathrm{dl}$ ), Twin pregnancy, and pregnancy complicated with abnormal uterine condition eg: - fibroids, placenta accrete

\section{Introduction}

Caesarean delivery is defined as the delivery of a foetus through surgical incisions made through lower abdominal wall (laparotomy) \& the uterine wall (hysterotomy). A LSCS is the most commonly used type of Caesarean section used today. It includes a transverse cut just above the edge of the bladder $\&$ results in less blood loss $\&$ is easier to repair then other types of Caesarean section. It may be transverse (the usual) or vertical in the following conditions. They are presence of lateral varicosities, constriction ring cut through it \& deeply engaged head. During LSCS most bleeding takes place from the angles of the incision \& forceps can be applied to control it. "Green Armitage forceps" are specially designed for this purpose. But the mother is at risk for increased bleeding (LSCS may result in twice the blood loss of a vaginal delivery) from the two incisions the placental attachment site $\&$ possible damage to uterine artery.

Pregnant woman who are healthy \& who have otherwise uncomplicated pregnancies should not routinely be offered blood grouping \& saving serum or cross-matching of blood before the Caesarean sections according to the current guidelines laid down by national institute of health \& care excellence (NICE). In Sri Lankan setup, grouping \& cross matching are done prior to each $\&$ every Caesarean sections without considering the presence or absence of risk factors even with less facilities available. It burdens the staff in blood bank with overwork \& increase the health expenditure incurred on the government. A scientific survey has not been conducted in Sri Lanka to address the issues related to this topic.

\section{Method}

This retrospective cross-sectional study was carried out at obstetric unit, General Hospital Matara, Sri Lanka. Our study population was all the mothers who underwent Elective \& Emergency caesarean sections at obstetric unit, General Hospital Matara, Sri Lanka. Our study sample was all the mothers who underwent Elective \& Emergency caesarean sections at obstetric unit, General 
Hospital Matara, Sri Lanka during one year (from $1^{\text {st }}$ of July 2013 to $30^{\text {th }}$ June 2014).

We collected our data from all the bed head tickets and other records available at blood bank belong to mothers who underwent Elective \& Emergency caesarean sections during above mention time period. A data extraction sheet used to retrieve relevant data from the records. The data was collected by investigators.

Ethical clearance for the study was obtained from the ethical committee of the Faculty of Medicine, University Of Ruhuna. Written permission to carry out the study was obtained from the administrative authorities of General Hospital Matara, Sri Lanka.

\section{Results}

Total number of mothers who underwent LSCS during $1^{\text {st }}$ of July 2013 to $30^{\text {th }}$ June 2014 was $3174.57 .5 \%$ of mothers underwent EL/LSCS \& 42.5\% underwent EM/ LSCS.

Total number of mothers who has transfused blood during LSCS was 59 \& as a percentage only $1.85 \%$. From that $3.38 \%$ was transfused during EL/LSCS \& $96.6 \%$ during EM/LSCS

According to our study, mothers who transfused during EL/LSCS were $50 \%$ due to uterine abnormality (lower segment large fibroid) \& 50\% due to placental abnormality (placenta accreata + past section).

When considering about EM/LSCS most of the mothers transfused due to Anemia it was $36.84 \%$. Second one was postpartum haemorrhage \& it was $26.31 \%$. Others are APH (Antipartum haemorrhage) 15.78\%,PIH (Pregnancy induce hypertension) $8.77 \%$,Placenta previa $7.01 \%$,Twin pregnancy $5.26 \%$

\section{Discussion}

According to our study, proportion of blood transfusion performed among mothers whose blood was grouped \& cross- matched for LSCS was $1.87 \%$. It means blood transfusion to cross matching ratio is low. In Sri Lanka grouped \& cross- matching are done prior to each \& every caesarean section without considering the presence or absence of risk factors. It burdens the staff at blood banks with over work \& increases the health expenditure to current government. According to our study all the mothers who underwent LSCS do not need cross matching before LSCS. It will reduce the work load on the staff at blood bank \& increases the quality of services rendered $\&$ it will cut down the expenditure on the healthcare supply \& this study may be helpful in making protocols for routine cross-matching before LSCS.

\section{References}

1. Obstetrics simplified-Diaa M.EL-Mowafi > caesarean section General foundation for medical Education \& Research Edited by Aldo campana, September 04/2008.

2. Lower segment caesarean section "primary surgery volume one": Non trauma. Prev. chapter 10 . The surgery of labour.

3. www.surgeryencyclopedia.com

4. Niceguideline2011NovemberCG132 https://www. nice.org.uk/guidance/cg132/.../caesarean.section update full guideline 